\title{
Retrospective analysis of palliative chemotherapy for the patients with bladder adenocarcinoma: Korean Cancer Study Group Genitourinary and Gynecology Cancer Committee
}

Moon Jin $\mathrm{Kim}^{1}$, Young Sam $\mathrm{Kim}^{2}$, Sung Yong $\mathrm{Oh}^{2}$, Suee Lee ${ }^{2}$, Young-Jin $\mathrm{Choi}^{3}$, Young Mi Seol${ }^{3}$, Min Jae Park 4 , Ki Hyang Kim5, Lee Chun Park ${ }^{6}$, Jung Hun Kang7, In-Gyu Hwang, , Soon Il Lee Seung Taek Lim ${ }^{10}$, Hyo Song Kim ${ }^{11}$, Ho Yeong Lim ${ }^{1}$, Sun Young Rha ${ }^{11}$, and Hyo-Jin Kim ${ }^{2}$

Department of Internal Medicine, ${ }^{1}$ Samsung Medical Center, Sungkyunkwan University School of Medicine, Seoul; ${ }^{2}$ Dong-A University Hospital, Busan; ${ }^{3}$ Pusan National University Hospital, Busan; ${ }^{4}$ Pusan National University Yangsan Hospital, Yangsan; ${ }^{5}$ Inje University Busan Paik Hospital, Busan; ${ }^{6}$ Kosin University College of Medicine, Busan; ${ }^{7}$ Gyeongsang National University Hospital, Jinju; ${ }^{8}$ ChungAng University College of Medicine, Seoul; ' Dankook University College of Medicine, Cheonan; ${ }^{10}$ Konyang University Hospital, Daejeon; ${ }^{11}$ Yonsei University College of Medicine, Seoul, Korea

\section{Received: June 7, 2015}

Revised : December 12, 2015

Accepted: December 31, 2015

\section{Correspondence to}

Sung Yong Oh, M.D.

Department of Internal Medicine,

Dong-A University Hospital, 26

Daesingongwon-ro, Seo-gu, Busan 49201, Korea

Tel: +82-51-240-2808

Fax: +82-51-246-5044

E-mail: drosy@dau.ac.kr
Background/Aims: Because of rarity, role of chemotherapy of bladder adenocarcinoma are still unidentified. Therefore, we performed a retrospective analysis of the clinical features and chemotherapy outcomes of bladder adenocarcinoma.

Methods: Eligible patients for this retrospective analysis were initially diagnosed with bladder adenocarcinoma and presented with a clinically no other primary site of origin. The collected data included age, gender, performance status, stage, hemoglobin, albumin, initial date of diagnosis, treatment modality utilized, response to treatment, presence of relapse, last status of patient, and last date of follow-up.

Results: We retrospectively reviewed 29 patients, who were treated with chemotherapy for bladder adenocarcinoma at 10 Korean medical institutions from 2004 to 2014 . The median age of patients was 58 years (range, 17 to 78 ) and $51.7 \%$ of the patients were female. Urachal adenocarcinoma was identified in 15 patients. Of 27 symptomatic patients, 22 experienced gross hematuria. Twelve patients were treated with 5-fluorouracil based chemotherapy, five were gemcitabine based, three were taxane and others. Thirteen of them achieved complete response (10.3\%) or partial response (34.5\%). Median progression-free survival (PFS) and overall survival (OS) for all patients were 10.6 months (95\% confidence interval [CI], 9.5 to 11.6 ) and 24.5 months ( $95 \%$ CI, 1.2 to 47.8 ), respectively. The cases of urachal adenocarcinoma exhibited worse tendency in PFS and OS $(p=0.024$ and $p=0.046$, respectively).

Conclusions: Even though bladder adenocarcinoma had been observed moderate effectiveness to chemotherapy, bladder adenocarcinoma is a highly aggressive form of bladder cancer. PFS and OS were short especially in urachal carcinoma.

Keywords: Urinary bladder; Adenocarcinoma; Drug therapy 


\section{INTRODUCTION}

Primary adenocarcinoma of the bladder is a malignant neoplasm derived from urothelium of the bladder showing histologically pure glandular differentiation [1]. Primary adenocarcinoma of the bladder is a rare malignancy, representing only $0.5 \%$ to $2.5 \%$ of all malignant bladder neoplasm $[2,3]$. This tumor is more common in males with approximate mean age of 50 to 60 years $[4,5]$. Generally, the tumor is very aggressive, with metastatic disease reported in up to $25 \%$ to $40 \%$ of patients at the time of diagnosis $[6,7]$. The pathogenesis of primary adenocarcinoma of the urinary bladder has not been fully elucidated. Intestinal metaplasia has been speculated to be a precursor lesion [8], largely because it is often seen in mucosa adjacent to primary adenocarcinoma of the urinary bladder. The diagnosis of a primary adenocarcinoma of the bladder should only be made when the carcinoma exhibits pure glandular differentiation. In many cases of adenocarcinoma of the bladder, the most important and the most difficult task is to differentiate these tumors from metastatic adenocarcinoma from other organs such as colon, lung, prostate, breast, and uterus. The most frequent secondary tumor involving the urinary bladder is adenocarcinoma of the colon, which morphologically resembles adenocarcinoma of the urinary bladder (enteric type). Because of its rarity, role of chemotherapy for advanced bladder adenocarcinoma is still questionable. Therefore, we performed a retrospective analysis of the clinical features and chemotherapy outcomes of advanced bladder adenocarcinoma to evaluate the clinical findings at presentation, treatment modality and outcome, overall survival (OS) and progression-free survival (PFS) and prognostic factors.

\section{METHODS}

\section{Patients}

We retrospectively reviewed the patients diagnosed with bladder adenocarcinoma at 10 Korean medical centers from 2005 to 2014. All the patients diagnosed adenocarcinoma through the tissue biopsy, and should have been treated at least one cycle of palliative chemotherapy for metastatic lesion. Adjuvant treatment after operation was excluded. And the patients who had favored primary colon, lung, or ovary lesion were excluded from the study.

Unified case report forms were provided to participating institutions. The collected data included age, gender, performance status, presenting symptom, stage, location of metastatic lesions, hemoglobin, initial date of diagnosis, and chemotherapy regimen utilized. We also obtained data regarding time to relapse, relationship with primary site and relapsing site, salvage treatment modality, and response and survival rates of salvage treatment. Responses were classified according to the Response Evaluation Criteria in Solid Tumors criteria. This study was approved by the Institutional Review Board of Dong-A University Hospital (DAUHIRB-14-108).

\section{Statistical analysis}

PFS was estimated from the date treatment began to the date when disease progression was recognized, or the date of the last follow-up visit, or the date of death. OS was calculated from the first day of diagnosis with metastatic or recurrent disease to the date of death or the last follow-up evaluation. OS and PFS were estimated using the Kaplan-Meier product-limit method. Survival rates were compared for statistical differences using log-rank analysis. The Cox regression model was used for multivariate analysis with factors that had been used in univariate (log rank) analysis of OS and PFS. All statistical analyses were performed using the IBM SPSS version 20.0 (IBM Co., Armonk, NY, USA). p value less than 0.05 was considered statistically significant and all $p$ values corresponded to two-sided significance tests.

\section{RESULTS}

\section{Patients' characteristics}

Between February 2005 and June 2014, a total of 29 patients from 10 different institutions in Korea were included. Diagnosis of bladder adenocarcinoma was made by examination of a partial or radical cystectomy specimen in 21 cases and by bladder biopsy in eight.

A list of patients and tumor characteristics is shown in Tables 1 and 2. The median age of patients was 58 years (range, 17 to 78 ) and $41.4 \%$ was over 60 years. Typical- 
Table 1. Patients' characteristics $(n=29)$

\begin{tabular}{|c|c|}
\hline Characteristic & No. (\%) \\
\hline \multicolumn{2}{|l|}{ Sex } \\
\hline Male & $14(48.3)$ \\
\hline Female & $15(51.7)$ \\
\hline \multicolumn{2}{|l|}{ Age, yr } \\
\hline Median (range) & $58(17-78)$ \\
\hline$\geq 60$ & $12(41.4)$ \\
\hline$<60$ & $17(58.6)$ \\
\hline \multicolumn{2}{|l|}{ Hemoglobin, g/dL } \\
\hline$\geq 12$ & $16(55.2)$ \\
\hline$<12$ & $13(44.8)$ \\
\hline \multicolumn{2}{|l|}{ Diagnosis } \\
\hline Urachal adenocarcinoma & $15(51.7)$ \\
\hline Adenocarcinoma nonspecific & $4(13.8)$ \\
\hline Mucinous adenocarcinoma & $4(13.8)$ \\
\hline Adenocarcinoma of enteric type & $2(6.9)$ \\
\hline Adenocarcinoma with signet-ring cell & $2(6.9)$ \\
\hline Adenocarcinoma, well differentiated & $1(3.4)$ \\
\hline Infiltrating adenocarcinoma & $1(3.4)$ \\
\hline \multicolumn{2}{|l|}{ Previous operation } \\
\hline Curative & $14(48.3)$ \\
\hline Palliative & $7(24.1)$ \\
\hline No operation & $8(25 \cdot 6)$ \\
\hline \multicolumn{2}{|l|}{ Metastatic presentation } \\
\hline Relapse & $14(48.3)$ \\
\hline Initial metastasis & $15(51.7)$ \\
\hline \multicolumn{2}{|l|}{ Organ of metastasis } \\
\hline Lung & $15(51.7)$ \\
\hline Peritoneum & $12(41.4)$ \\
\hline Ovary & $6(20.7)$ \\
\hline Lymph node & $4(13.8)$ \\
\hline Bone & $3(10.3)$ \\
\hline Bladder/ureter & $2(6.9)$ \\
\hline Colon/rectum & $2(6.9)$ \\
\hline Liver & $1(3.4)$ \\
\hline
\end{tabular}

ly, $51.7 \%$ of the patients were female. Of 27 symptomatic patients, 22 and six patients experienced gross hematuria and palpable mass, respectively. Other presenting symptoms included abdominal distension, dysuria/ frequency, and abdominal pain. The median level of hemoglobin was $12.1 \mathrm{~g} / \mathrm{dL}$ (range, 6.9 to 15.4). The most
Table 2. Symptoms of advanced bladder adenocarcinoma $(\mathbf{n}=\mathbf{2 9})$

\begin{tabular}{lc}
\hline Variable & No. $(\%)$ \\
\hline Hematuria & $22(75.9)$ \\
Palpable mass & $6(20.7)$ \\
Abdominal distension & $5(17.2)$ \\
Dysuria/frequency & $4(13.8)$ \\
Abdominal pain & $3(10.3)$ \\
Fever & $1(3.4)$ \\
Vaginal spotting & $1(3.4)$ \\
Dyspnea & $1(3.4)$ \\
No subjective symptom & $2(6.9)$ \\
\hline
\end{tabular}

frequent pathological diagnosis was urachal adenocarcinoma (48.3\%) followed by nonspecific adenocarcinoma and mucinous adenocarcinoma (13.8\%), adenocarcinoma of enteric type and adenocarcinoma with signet ring cell (6.9\%), well-differentiated adenocarcinoma (3.4\%), and infiltrating adenocarcinoma (3.4\%). Patients were initially presented with metastatic lesion or in relapsed state after definitive treatment. Eight patients (25.6\%) had no history of prior operation, and 21 patients had previously received curative (48.3\%) or palliative (24.1\%) operation. Fifteen patients presented with initially distance metastatic lesions. Twelve patients had two or more metastatic sites of the lung (51.7\%), peritoneum (41.4\%), ovary (20.7\%), and lymph nodes (13.8\%) as the most common sites.

\section{Palliative chemotherapy and response}

Patients were treated with several variable chemotherapy regimens (Table 3). Usual chemotherapeutic regimens for urothelial cell carcinoma-GP (gemcitabine + cisplatin), MVAC (methotrexate + vinblastine + adriamycin + cisplatin), MVP (methotrexate + vinblastine + cisplatin)—were used in nine patients. Among them, partial response (PR) was observed in four patients.

The 5-fluorouracil (5-FU) based chemotherapeutic regimens-FP (5-FU + cisplatin), FAP (5-FU + adriamycin + cisplatin), FOLFOX (oxaliplatin + leucovorin + 5-FU), FEP (5-FU + epirubicine + cisplatin)-were used in 12 patients. Of the 12 patients, PR was observed in five patients. The three patients treated with taxane and platinum combination regimen experienced two CRs. 
Table 3. Chemotherapy result of advanced bladder adenocarcinoma $(\mathbf{n}=\mathbf{2 9})$

\begin{tabular}{|c|c|c|c|c|c|c|}
\hline Chemotherapy & Number & $\mathrm{CR}^{\mathrm{a}}$ & $\mathrm{PR}^{\mathrm{a}}$ & $\mathrm{SD}$ & $\mathrm{PD}$ & $\mathrm{NE}$ \\
\hline GP & 5 & & 2 & 1 & & 2 \\
\hline MVAC & 3 & & 2 & & 1 & \\
\hline MVP & 1 & & & & 1 & \\
\hline FP & 7 & & 3 & 3 & & 1 \\
\hline FOLFOX & 1 & & 1 & & & \\
\hline FAP & 2 & & 1 & & & 1 \\
\hline FEP & 1 & & & 1 & & \\
\hline 5-FU single & 1 & & & 1 & & \\
\hline EP & 3 & 1 & & 1 & & 1 \\
\hline Taxane/platinum & 3 & 2 & & & 1 & \\
\hline Pemetraxed & 1 & & 1 & & & \\
\hline Sunitinib & & & & & 1 & \\
\hline
\end{tabular}

CR, complete response; $\mathrm{PR}$, partial response; SD, stable disease; PD, progression of disease; NE, not evaluable; GP, gemcitabine + cisplatin; MVAC, methotrexate + vinblastine + adriamycin + cisplatin; MVP, methotrexate + vinblastine + cisplatin; FP, 5-FU + cisplatin; FOLFOX, oxaliplatin + leucovorin + 5-FU; FAP, 5-FU + adriamycin + cisplatin; FEP, 5-FU + epirubicine + cisplatin; 5-FU, 5-fluorouracil; EP, etoposide + cisplatin.

${ }^{\mathrm{a}} \mathrm{CR}+\mathrm{PR}=13(44.8 \%)$.

\section{Survivals and prognostic factor analysis}

During the median follow-up period of 12.7 months (range, 1.6 to 79.7 ) progression was seen in 21 patients, while 13 patients $(25.7 \%)$ died. Median PFS and OS for all patients were 10.6 months (95\% confidence interval [CI], 9.5 to 11.6) and 24.5 months (95\% CI, 1.2 to 47.8 ), respectively (Fig. 1). Patients with nonurachal adenocarcinomas had a better median PFS of 20.6 months when compared with 10.4 months of patients with urachal adenocarcinomas $(p=0.024)$ (Fig. 2$)$. In median OS, nonurachal adenocarcinomas patients who did not reached median OS were better than 16.3 months of urachal adenocarcinomas $(p=0.046)$ (Fig. 3$)$. The clinical factors predicting reduced in PFS and OS was subtype of adenocarcinoma especially in urachal adenocarcinoma (Table 4).

\section{DISCUSSION}

Our study evaluated the efficacy of palliative chemotherapy for adenocarcinoma of bladder and until date, it is one of the largest reports on the efficacy of palliative chemotherapy for the treatment of advanced bladder adenocarcinoma, even though the number of patients was relatively small and retrospective analysis.

Usually it has been reported that male is predom-

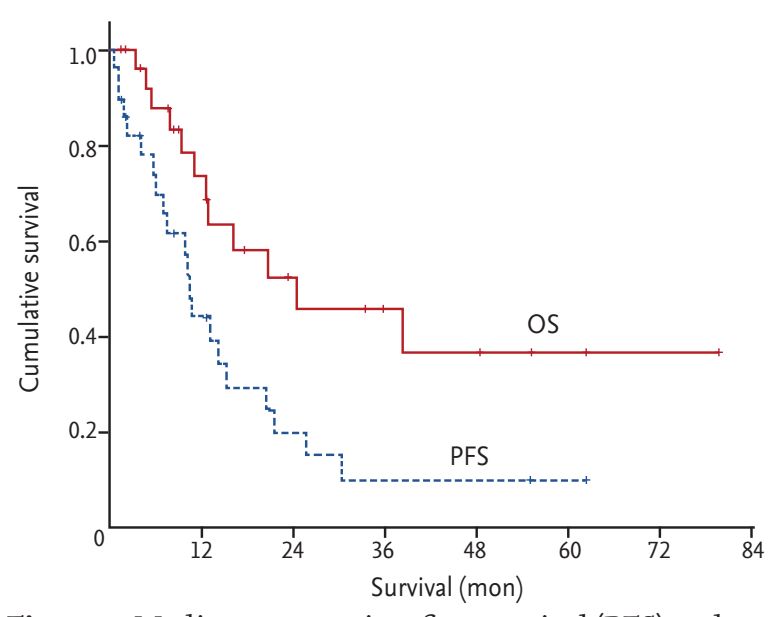

Figure 1. Median progression-free survival (PFS) and overall survival (OS) for all patients were 10.6 months (95\% confidence interval [CI], 9.5 to 11.6 ) and 24.5 months (95\% CI, 1.2 to 47.8$)$.

inant in bladder adenocarcinoma [4,5,9]. Because of small study population, numbers of female was a little bite larger than male in this study. Nontransitional cell urothelial carcinoma including adenocarcinoma, squamous cell carcinoma, or small cell carcinoma is an uncommon tumor and accounts for only $5 \%$ to $10 \%$ of urothelial cancers $[10,11]$. Thus, the clinical experience 


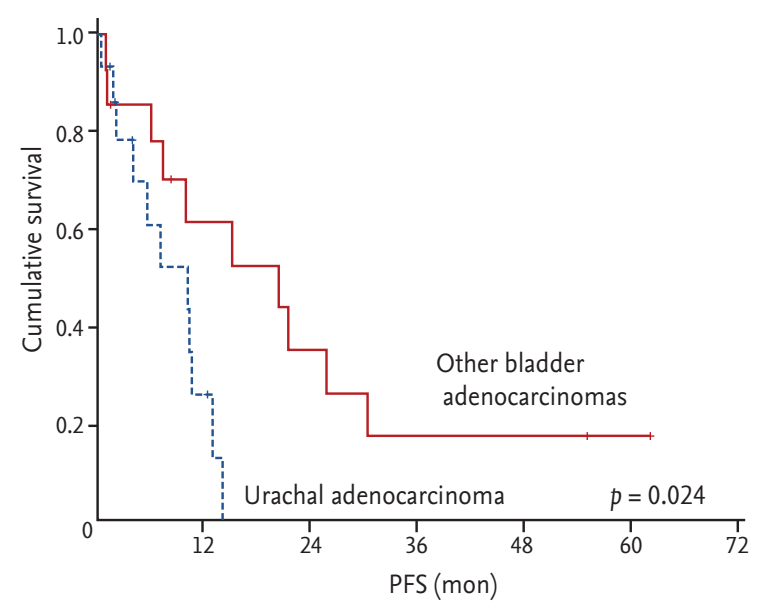

Figure 2. Patients with nonurachal adenocarcinomas had a better median progression-free survival (PFS) of 20.6 months when compared with 10.4 months in patients with urachal adenocarcinomas $(p=0.024)$.

with chemotherapy is limited, and to date, treatment recommendations have largely been derived based on case reports and small series [11-14]. Galsky et al. [12] reported the chemotherapeutic outcomes for 20 patients with nontransitional cell carcinoma of the urothelial tract (11 patients with adenocarcinomas, eight patients with squamous cell carcinomas, and one patient with small cell carcinoma), using a chemotherapeutic regimen consisting of palitaxel, cisplatin, and ifosfamide with mesna. In the reported study, seven patients had a CR or PR, with an objective response rate of $35 \%$ (36\% in adenocarcinoma). The median OS was 24.8 months in patients with adenocarcinomas and 9 months in those with squamous cell carcinomas. A study by Hong et al. [4] showed an objective response rate of $36 \%$ to various first-line chemotherapeutic regimens and a median OS of 47 months in 14 patients with adenocarcinoma. Siefker-Radtke [13] reported median survival from recognition of metastatic disease as 24 months in 26 patients in whom metastases ultimately developed. Chemotherapy with various regimens for metastatic disease produced only four significant responses, including three of nine patients treated with 5-FU and cisplatin containing regimens. As per our result, $44.8 \%$ of overall response rate was higher and 24.5 months of median survival was comparable with prior studies (Table 5).

When we choose the chemotherapeutic regimen, we don't have any evidence based consensus regimen for bladder adenocarcinoma. Transitional cell cancers

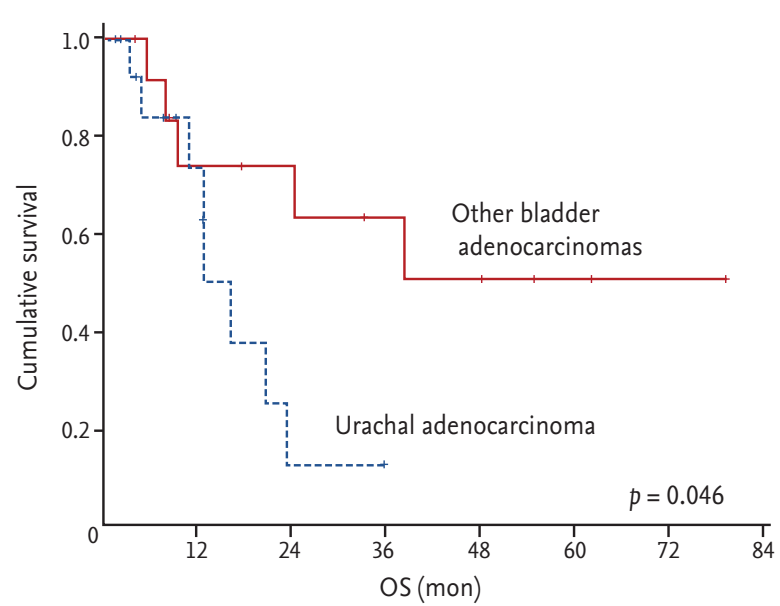

Figure 3. Patients with nonurachal adenocarcinomas had a better median overall survival (OS) when compared with 16.3 months in patients with urachal adenocarcinomas $(p=0.046)$.

(TCCs) are treated with gemcitabine-platinum combination, MVAC, or MVP regimens, while adenocarcinoma is usually treated with 5-FU based combination chemotherapy. In this study, TCC focused treatment and adenocarcinoma focused treatments were effective and no differences in PFS and OS. Considering an objective response rate of $39 \%$ to $65 \%$ to the MVAC or gemcitabine-platinum regimens in advanced transitional cell carcinoma [15-17], the objective response of palliative chemotherapy in advanced bladder adenocarcinoma is comparatively lower than transitional cell carcinoma. However, the OS of 24.5 months of this study showed comparable survival results with previous studies regarding transitional cell carcinoma patients $[18,19]$.

We observed that patients with nonurachal adenocarcinomas had a better median PFS of 20.6 months when compared with 10.4 months in patients with urachal adenocarcinomas $(p=0.024)$ in the metastatic palliative chemotherapy setting. However, it remains unclear whether the survival of patients with urachal adenocarcinoma differs from that of patients with nonurachal adenocarcinomas itself. Wright et al. [20] found that urachal cancer had a significantly better prognosis than nonurachal cancer. Compared to those with nonurachal tumors, patients with urachal adenocarcinoma were more likely to be younger (median age, 56 years vs. 69 years, $p<0.001$ ) and female ( $45 \%$ vs. $36 \%, p=0.02$ ). Urachal lesions were less likely to be high grade (35\% vs. $66 \%, p<0.001$ ), but more likely to involve distant me- 
Table 4. Prognostic factor analysis of survivals

\begin{tabular}{|c|c|c|c|c|}
\hline \multirow{2}{*}{ Variable } & \multicolumn{2}{|c|}{ PFS } & \multicolumn{2}{|c|}{ OS } \\
\hline & Median, mon & $p$ value $^{a}$ & Median, mon & $p$ value $^{a}$ \\
\hline Sex & & 0.755 & & 0.382 \\
\hline Male & 10.1 & & 16.3 & \\
\hline Female & 13.2 & & $-b$ & \\
\hline Age, yr & & 0.205 & & 0.374 \\
\hline$\geq 60$ & 10.4 & & 12.9 & \\
\hline$<60$ & 13.2 & & $24 \cdot 5$ & \\
\hline Initial status & & 0.654 & & 0.725 \\
\hline Relapsed & 10.1 & & $23 \cdot 5$ & \\
\hline Metastatic & 13.2 & & 38.4 & \\
\hline Hemoglobin, g/dL & & 0.101 & & 0.835 \\
\hline$\geq 12$ & 14.3 & & 23.5 & \\
\hline$<12$ & 10.4 & & 12.9 & \\
\hline Hematuria & & 0.074 & & 0.478 \\
\hline$(+)$ & 10.6 & & $23 \cdot 5$ & \\
\hline$(-)$ & 6.1 & & $-\mathrm{b}$ & \\
\hline Lung involvement & & 0.905 & & 0.821 \\
\hline$(+)$ & 10.8 & & $23 \cdot 5$ & \\
\hline$(-)$ & 10.6 & & 16.3 & \\
\hline Peritoneal seeding & & 0.117 & & 0.647 \\
\hline$(+)$ & 7.2 & & 20.7 & \\
\hline$(-)$ & $14 \cdot 3$ & & 38.4 & \\
\hline 5-FU included regimens & & $0.15^{8}$ & & 0.486 \\
\hline$(+)$ & 10.1 & & 16.3 & \\
\hline$(-)$ & $14 \cdot 3$ & & $23 \cdot 5$ & \\
\hline Urinary tract regimens ${ }^{c}$ & & 0.827 & & 0.545 \\
\hline$(+)$ & $14 \cdot 3$ & & $23 \cdot 5$ & \\
\hline$(-)$ & 10.4 & & 20.7 & \\
\hline Subtype & & 0.024 & & 0.046 \\
\hline Urachal adenocarcinoma & 10.4 & & 16.3 & \\
\hline The others & 20.6 & & $-\mathrm{b}$ & \\
\hline
\end{tabular}

PFS, progression-free survival; OS, overall survival; 5-FU, 5-fluorouracil.

${ }^{a}$ By log-rank test for univariate analysis.

${ }^{\mathrm{b}} \mathrm{Did}$ not reached median value.

${ }^{\mathrm{c}}$ Gemcitabine/cisplatin, MVAC (methotrexate + vinblastine + adriamycin + cisplatin), or MVP (methotrexate + vinblastine + cisplatin).

tastases (30\% vs. 15\%, $p<0.001)$. While Mostofi et al. [21] reported that urachal adenocarcinomas were associated with a significantly worse prognosis. The 17 tumors that were probably urachal in origin were characterized clinically by a more unfavorable course, all but three of the patients having died in less than 5 years. The poor prognosis of the urachal tumor is believed to be due to incomplete removal, for these tumors tend to spread along the remnants of the pre-existing urachal tract, not only in the bladder wall but in the anterior abdominal wall as well. Dandekar et al. [22] and Zhang et al. [9] stated the difference between urachal adenocarcinoma and 
Table 5. Comparison with prior chemotherapy for bladder adenocarcinoma

\begin{tabular}{|c|c|c|c|c|c|}
\hline Study & No. of patients & Regimen & ORR, \% & PFS, mon & OS, mon \\
\hline Hong et al. (2009) [4] & 14 & $\begin{array}{l}\text { GP, FP, TP, MVAC, MVP, VIP, TC, EP, } \\
\text { BOMP, VI, paclitaxel }\end{array}$ & 36 & 8 & 47 \\
\hline Galsky et al. (2007) [12] & 11 & TIP & 36 & NR & 24.8 \\
\hline Siefker-Radtke (2012) [13] & 26 & $\begin{array}{l}\text { 5-FU, } \alpha \text {-interferon, cisplatin, MVAC, } \\
\text { paclitaxel based, ifosfamide based }\end{array}$ & $15 \cdot 4$ & NR & 24 \\
\hline Present study & 29 & GP, MVAC, MVP, FP, FAP, FOLFOX, TP & 44.8 & 10.6 & $24 \cdot 5$ \\
\hline
\end{tabular}

ORR, overall response rate; PFS, progression-free survival; OS, overall survival; GP, gemcitabine + cisplatin; FP, 5-FU + cisplatin; TP, paclitaxel + cisplatin; MVAC, methotrexate + vinblastine + adriamycin + cisplatin; MVP, methotrexate + vinblastine + cisplatin; VIP, etoposide + ifosfamide + cisplatin; TC, paclitaxel + carboplatin; EP, etoposide + cisplatin; BOMP, bleomycin + vincristine + mitomycin + cisplatin; VI, etoposide + ifosfamide; NR, not reported; TIP, paclitaxel + ifosfamide + cisplatin; 5-FU, 5-fluorouracil; FAP, 5-FU + adriamycin + cisplatin; FOLFOX, oxaliplatin + leucovorin + 5-FU.

nonurachal adenocarcinoma as not significant. These different results between studies about prognostic value of urachal and nonurachal adenocarcinoma might be depending on included different proportion of early stage, treatment option (operability), and comorbidity of patients.

This study has several limitations. This study was a retrospective analysis. Therefore it could have possibility of selection bias, insufficiency of medical records, and heterogeneity of treatment. The number of patients enrolled in the study was too small to evaluate efficacy of chemotherapy and analyze prognostic factors. Nevertheless, considering the relatively uncommon incidence of the disease, this is one of the largest data sets reporting clinical results of chemotherapy and could have an importance of histological types as a prognostic factor in advanced bladder adenocarcinoma.

Prospective trials are warranted to make a clinically meaningful improvement in palliative chemotherapy in the treatment of advanced bladder adenocarcinomas.

\section{KEY MESSAGE}

1. Primary adenocarcinoma of the bladder is a rare malignancy.

2. Advanced bladder adenocarcinoma was also a highly aggressive form like a transitional cell bladder carcinoma.

3. Nonurachal adenocarcinomas had a better median progression-free survival and overall survival compared with urachal adenocarcinomas.

\section{Conflict of interest}

No potential conflict of interest relevant to this article was reported.

\section{Acknowledgments}

This paper was supported by the Dong-A University Research Fund.

\section{REFERENCES}

1. Zhong M, Gersbach E, Rohan SM, Yang XJ. Primary adenocarcinoma of the urinary bladder: differential diagnosis and clinical relevance. Arch Pathol Lab Med 2013;137:371-381.

2. Bates AW, Baithun SI. Secondary neoplasms of the bladder are histological mimics of nontransitional cell primary tumours: clinicopathological and histological features of 282 cases. Histopathology 2000;36:32-40.

3. Bardales RH, Pitman MB, Stanley MW, Korourian S, Suhrland MJ. Urine cytology of primary and secondary urinary bladder adenocarcinoma. Cancer 1998;84:335-343.

4. Hong JY, Choi MK, Uhm JE, et al. Palliative chemotherapy for non-transitional cell carcinomas of the urothelial tract. Med Oncol 2009;26:186-192.

5. Zhang J, Wu J. Options for diagnosis and treatment of urachal carcinoma. Asia Pac J Clin Oncol 2013;9:117-122.

6. Akamatsu S, Takahashi A, Ito M, Ogura K. Primary signet-ring cell carcinoma of the urinary bladder. Urology 2010;75:615-618.

7. Romics I, Szekely E, Szendroi A. Signet-ring cell carcinoma arising from the urinary bladder. Can J Urol 
2008;15:4266-4268.

8. Smith AK, Hansel DE, Jones JS. Role of cystitis cystica et glandularis and intestinal metaplasia in development of bladder carcinoma. Urology 2008;71:915-918.

9. Zhang H, Jiang H, Wu Z, Fang Z, Fan J, Ding Q. Primary adenocarcinoma of the urinary bladder: a single site analysis of 21 cases. Int Urol Nephrol 2013;45:107-111.

10. Dahm P, Gschwend JE. Malignant non-urothelial neoplasms of the urinary bladder: a review. Eur Urol 2003;44:672-681.

11. Manunta A, Vincendeau S, Kiriakou G, Lobel B, Guille F. Non-transitional cell bladder carcinomas. BJU Int 2005;95:497-502.

12. Galsky MD, Iasonos A, Mironov S, et al. Prospective trial of ifosfamide, paclitaxel, and cisplatin in patients with advanced non-transitional cell carcinoma of the urothelial tract. Urology 2007;69:255-259.

13. Siefker-Radtke A. Urachal adenocarcinoma: a clinician's guide for treatment. Semin Oncol 2012;39:619-624.

14. Miyata Y, Sagara Y, Matsuo T, et al. Response of recurrent urachal cancer to gemcitabine and cisplatin therapy: a case report and literature review. Anticancer Res 2011;31:23352338.

15. Logothetis CJ, Dexeus FH, Finn L, et al. A prospective randomized trial comparing MVAC and CISCA chemotherapy for patients with metastatic urothelial tumors. J
Clin Oncol 1990;8:1050-1055.

16. Bellmunt J, von der Maase H, Mead GM, et al. Randomized phase III study comparing paclitaxel/cisplatin/ gemcitabine and gemcitabine/cisplatin in patients with locally advanced or metastatic urothelial cancer without prior systemic therapy: EORTC Intergroup Study 30987. J Clin Oncol 2012;30:1107-1113.

17. De Santis M, Bellmunt J, Mead G, et al. Randomized phase II/III trial assessing gemcitabine/carboplatin and methotrexate/carboplatin/vinblastine in patients with advanced urothelial cancer who are unfit for cisplatin-based chemotherapy: EORTC study 30986. J Clin Oncol 2012;30:191-199.

18. Ismaili N, Amzerin M, Flechon A. Chemotherapy in advanced bladder cancer: current status and future. J Hematol Oncol 2011;4:35.

19. Carballido EM, Rosenberg JE. Optimal treatment for metastatic bladder cancer. Curr Oncol Rep 2014;16:404.

20. Wright JL, Porter MP, Li CI, Lange PH, Lin DW. Differences in survival among patients with urachal and nonurachal adenocarcinomas of the bladder. Cancer 2006;107:721-728.

21. Mostofi FK, Thomson RV, Dean AL Jr. Mucous adenocarcinoma of the urinary bladder. Cancer 1955;8:741-758.

22. Dandekar NP, Dalal AV, Tongaonkar HB, Kamat MR. Adenocarcinoma of bladder. Eur J Surg Oncol 1997;23:157-160. 